\title{
AN ANOMALOUS QUIESCENT STELLAR MASS BLACK HOLE
}

\author{
Mark T. Reynolds and Jon M. Miller \\ Department of Astronomy, University of Michigan, 500 Church Street, Ann Arbor, MI 48109, USA; markrey@umich.edu \\ Received 2011 March 28; accepted 2011 May 4; published 2011 May 19
}

\begin{abstract}
We present the results of a 40 ks Chandra observation of the quiescent stellar mass black hole GS 1354-64. A total of 266 net counts are detected at the position of this system. The resulting spectrum is found to be consistent with the spectra of previously observed quiescent black holes, i.e., a power law with a photon index of $\Gamma \sim 2$. The inferred luminosity in the $0.5-10 \mathrm{keV}$ band is found to lie in the range $0.5-6.5 \times 10^{34} \mathrm{erg} \mathrm{s}^{-1}$, where the uncertainty in the distance is the dominant source of this large luminosity range. Nonetheless, this luminosity is over an order of magnitude greater than that expected from the known distribution of quiescent stellar mass black hole luminosities and makes GS 1354-64 the only known stellar mass black hole to disagree with this relation. This observation suggests the possibility of significant accretion persisting in the quiescent state.
\end{abstract}

Key words: accretion, accretion disks - black hole physics - X-rays: binaries

Online-only material: color figures

\section{INTRODUCTION}

Observations at optical and infrared wavelengths have allowed us to measure the mass of 19 Galactic and four extragalactic stellar mass black holes $\left(M_{\mathrm{BH}} \sim 10-20 M_{\odot}\right.$; McClintock \& Remillard 2006; Orosz et al. 2007; Silverman \& Filippenko 2008; Crowther et al. 2010; Corral-Santana et al. 2011). The presence of a black hole is inferred whenever the mass of the compact object is dynamically constrained to be greater than $3 M_{\odot}$ (Rhoades \& Ruffini 1974; Kalogera \& Baym 1996). This does not confirm the compact object to be a classical black hole and these observations could be interpreted to agree with more exotic physics (see Narayan \& McClintock 2008 for details). In order to confirm that the objects above are indeed black holes, one is required to detect a unique characteristic of such objects, i.e., the event horizon.

X-ray observations of accreting black holes have revealed a number of distinct accretion regimes, which correlate in a broad sense with luminosity of the source. The very high, soft, and hard states (hereafter VHS, SS, and HS) are the primary active accretion states observed in X-ray backgrounds (XRBs). At luminosities below that observed in the HS state the system is said to be in the quiescent state. The quiescent state is characterized as an extremely faint state $\left(L_{x} \leqslant 10^{33.5} \mathrm{erg} \mathrm{s}^{-1}\right)$, with an X-ray spectrum that is distinctly non-thermal $(\Gamma=$ 1.5-2.2; McClintock \& Remillard 2006; Corbel et al. 2006).

The quiescent X-ray emission from black hole binaries (BHBs) is not consistent with expectations from standard accretion disk theory (e.g., McClintock et al. 1995). The current paradigm for understanding the X-ray emission from quiescent BHBs involves a standard thin disk, which transforms to a quasispherical inner flow at a distance of $\sim 10^{3}-10^{4}$ Schwarzschild radii from the black hole. The inner flow could consist of an advection-dominated accretion flow (ADAF; see Narayan \& McClintock 2008, and references therein). In an ADAF the energy, released via viscous dissipation, remains in the accreting gas rather than being radiated away. As a result, most of the energy is advected with the accretion flow resulting in only a small percentage of the energy being radiated by the gas before it reaches the compact object. The radiative efficiency of such a flow is expected to be in the range $\sim 0.01 \%-1 \%$. In comparison, a standard thin disk is expected to have an efficiency an order of magnitude greater. The ADAF model has been successfully applied to observations of a number of quiescent and hard state BHBs, e.g., Narayan et al. (1996). The ADAF solution is not limited to stellar mass black holes and has also been successfully used to model the emission from a number of supermassive black holes, e.g., see Yuan et al. (2003) for an ADAF fit to the spectrum of the least luminous known black hole Sgr A* $\left(L_{x} \sim 10^{-9} L_{\text {Edd }}\right)$.

However, the ADAF solution also allows outflows as emphasized by Blandford \& Begelman (1999). In recent years, jets/ outflows have been recognized as a ubiquitous feature associated with the process of accretion on the largest and smallest scales, e.g., active galactic nuclei, XRBs. Fender et al. (2003) proposed an alternative scheme, whereby at low luminosity ( $\left.L_{x} \lesssim 10^{-4} L_{\text {Edd }}\right)$ BHs should enter a "jet-dominated" state, in which the majority of the accretion power drives a radiatively inefficient jet. The detection of the black hole A0620-00 at radio wavelengths may support this (Gallo et al. 2006), although the absence of a discernible jet in Sgr A* is problematic (Narayan $\&$ McClintock 2008). However, the detection of frequency dependent time lags in the radio flares from Sgr A* (Yusef-Zadeh et al. 2009), combined with the stratified nature of the radio and millimeter-wave emission (Doeleman et al. 2008) strongly suggest an unbound, mildly relativistic outflow. Recent work envisages the X-ray flux from a quiescent system to be a combination of an outflow/jet and an inner advective region, e.g., Yuan et al. (2009).

With the advent of the Chandra and XMM-Newton X-ray observatories, detailed observations of quiescent black hole and neutron star binary systems have become possible, i.e., $L_{x} \lesssim 10^{-6} L_{\text {Edd }}$. As first pointed out in Narayan et al. (1997) and Garcia et al. (1998), the observed luminosities of the quiescent BHs are systematically fainter than NSs. For a black hole the energy stored in the flow is advected across the event horizon, whereas for a neutron star the material strikes the solid surface where it is re-radiated (Garcia et al. 2001; Kong et al. 2002). Subsequent observations have confirmed this picture (Sutaria et al. 2002; Hameury et al. 2003; Tomsick et al. 2003; McClintock et al. 2004; Corbel et al. 2006; Gallo et al. 2008).

GS 1354-64 was discovered by Ginga in 1987, where it displayed an X-ray spectrum dominated by a soft blackbody disk component, consistent with that of the then known BHBs 
Table 1

Broadband Continuum Fit Parameters

\begin{tabular}{|c|c|c|c|c|c|c|c|}
\hline Model & $\begin{array}{c}N_{\mathrm{H}} \\
\left(10^{22} \mathrm{~cm}^{-2}\right)\end{array}$ & $\Gamma$ & $\begin{array}{c}\mathrm{kT} \\
(\mathrm{keV})\end{array}$ & $\begin{array}{c}f_{\text {xabs }}(0.5-10.0 \mathrm{keV}) \\
\left(10^{-14} \mathrm{erg} \mathrm{s}^{-1} \mathrm{~cm}-2\right)\end{array}$ & $\begin{array}{c}f_{x}(0.5-10.0 \mathrm{keV}) \\
\left(10^{-14} \operatorname{erg~s}^{-1} \mathrm{~cm}-2\right)\end{array}$ & $\begin{array}{c}L_{x}(25 \mathrm{kpc}, 61 \mathrm{kpc}) \\
\left(10^{33} \mathrm{erg} \mathrm{s}^{-1}\right)\end{array}$ & $\chi^{2} / v$ \\
\hline pha (po) & $0.9_{-0.4}^{+0.45}$ & $2.2_{-0.4}^{+0.6}$ & $\cdots$ & $7.69_{-0.7}^{+0.9}$ & $14.5_{-1.3}^{+1.7}$ & $7.65_{-0.68}^{+0.90}, 64.6_{-5.8}^{+7.5}$ & $14.3 / 10$ \\
\hline pha(bremss) & $0.58_{-0.28}^{+0.34}$ & $\ldots$ & $5.1_{-2.4}^{+7.3}$ & $7.4_{-0.7}^{+0.9}$ & $10.7_{-1.2}^{+1.0}$ & $5.65_{-0.64}^{+0.52}, 47.6_{-5.3}^{+4.5}$ & $15.7 / 10$ \\
\hline
\end{tabular}

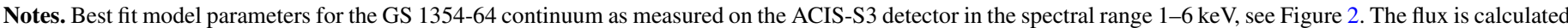

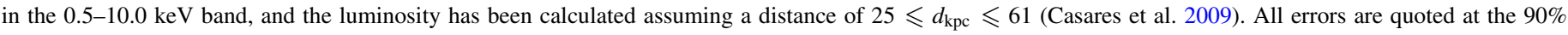
confidence level.

in the soft state (Makino 1987; Kitamoto et al. 1990). A second outburst was observed from this system in 1997 by RXTE, though on this occasion the system remained in the low-hard state throughout the outburst (Brocksopp et al. 2001). The black hole nature of the primary has been dynamically confirmed by Casares et al. (2004, 2009), who found the system to comprise of a G0-5 III mass donor in a $\sim 2.5$ day orbit around the black hole. The measured radial velocity $\left(\sim 279 \mathrm{~km} \mathrm{~s}^{-1}\right)$ in combination with the absence of any X-ray eclipses sets a secure lower limit for the mass of the black hole of $M_{x} \geqslant 7.6 \pm 0.7 M_{\odot}$. GS 135464 is found to lie at a large distance, where $25 \mathrm{kpc} \leqslant d \leqslant 61 \mathrm{kpc}$, and the upper limit is obtained by requiring the 1987 outburst to be sub-Eddington. This makes GS 1354-64 the most distant known Galactic black hole. The field containing GS 1354-64 has not been previously observed by either XMM-Newton or Chandra. The only X-ray imaging of this field was carried out by ROSAT as part of the all sky survey (Voges et al. 1999), where only a weak upper limit exists, $f_{x} \leqslant 5.6 \times 10^{-13} \mathrm{erg} \mathrm{s}^{-1} \mathrm{~cm}^{-2}$.

In this Letter, we describe an observation of the quiescent Galactic stellar mass BHB GS 1354-64 with the Chandra X-ray Observatory. We describe the observations and extraction of source spectra and light curves, these data are discussed in the context of models for the quiescent accretion flow.

\section{OBSERVATIONS}

GS 1354-64 was observed by Chandra for $40 \mathrm{ks}$ on 2010 October 1 (MJD 55470, obsid: 12471, PI: Reynolds), where it was placed on the back-illuminated ACIS-S3 detector, which was operated in VFAINT mode. A significant point source is detected consistent with the known position of GS 1354-64 (Brocksopp et al. 2001). As the I3, S1-S4 detectors were active during this observation, a number of serendipitous point sources are also detected. These sources (31 in total) and their properties will be described in a separate publication.

The GS 1354-64 spectrum was extracted from a 2".5 region with psextract, which also generated the appropriate response files. A background spectrum was extracted from a neighboring source free region on the detector. We detect 266 net counts consistent with the known position of GS 1354-64. The source and background light curves were extracted using dmextract and binned using lcurve. Inspection of the background light curve reveals an absence of any significant flares during the observation. The background subtracted light curve is displayed in Figure 1, where it has been divided into $5 \mathrm{ks}$ bins. Variability of a factor of $2 \times$ is observed on kilo-second timescales, e.g., the count rate is observed to increase from an average of $\sim 0.006 \mathrm{ct} \mathrm{s}^{-1}$ to $\sim 0.012 \mathrm{ct} \mathrm{s}^{-1}$ at the $30 \mathrm{ks}$ mark.

All data reduction and analysis takes place within the CIAO 4.2 and HEASOFT 6.6.2 environment, which includes FTOOLS 6.6 and XSPEC 12.5.0AJ. The latest versions of the relevant Chandra CALDB files are also used.

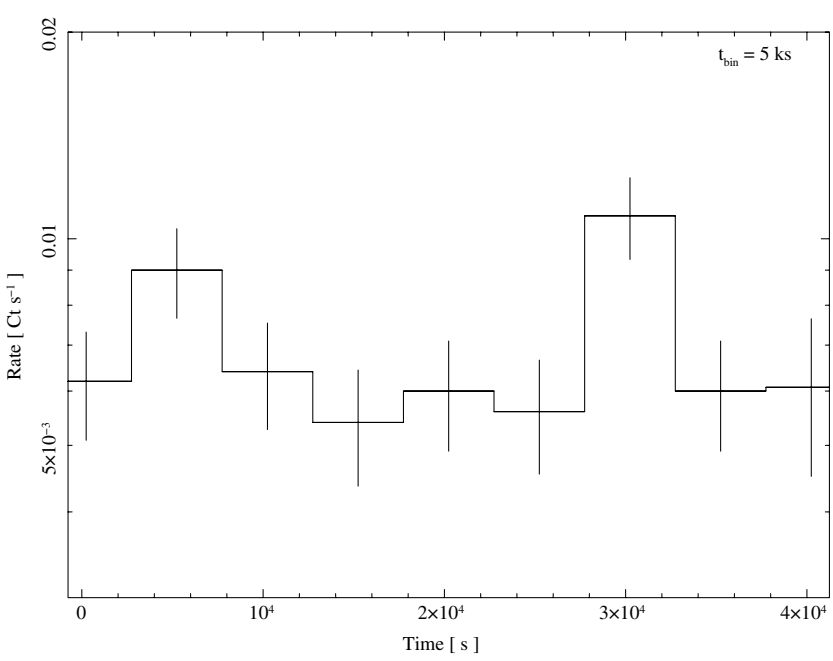

Figure 1. GS 1354-64 background subtracted light curve as observed by Chandra, where the light curve has been divided into $5 \mathrm{ks}$ bins. Variability of a factor of two is observed on kilo-second timescales.

\section{ANALYSIS}

The relatively large number of counts detected facilitates basic spectral fitting. The spectrum was binned such that each bin contained 20 counts using grppha, providing useful data in the spectral range $1-6 \mathrm{keV}$. We model the spectrum using two models for the continuum (1) a power law (pha*po) and (2) bremsstrahlung (pha*brem). The results of the model fits are displayed in Table 1 . The best fit power-law spectral index of $\Gamma \sim 2.1$ (see Figure 2) and the bremsstrahlung temperature of $T_{B} \sim 5 \mathrm{keV}$ are consistent with previous observations of quiescent stellar mass black holes, e.g., Kong et al. (2002) and Bradley et al. (2007). We measure an absorbed flux of $\sim 7.7(7.4) \times 10^{-14} \mathrm{erg} \mathrm{s}^{-1} \mathrm{~cm}^{-2}$ in the $0.5-10.0 \mathrm{keV}$ band for the power-law(bremsstrahlung) model. This corresponds to an unabsorbed flux of approximately $\sim 1.5(1.1) \times 10^{-13} \mathrm{erg} \mathrm{s}^{-1} \mathrm{~cm}^{-2}$ in the same energy range, see Table 1 for details.

In Figure 3, we plot contours of power-law index and bremsstrahlung temperature versus extinction for each of the models above. The uncertainty regions are large, consistent with the small energy range covered by the data. Nonetheless, we see that the power-law model favors significantly larger values for the extinction $N_{\mathrm{H}} \sim 9 \times 10^{21} \mathrm{~cm}^{-2}$. For comparison, Kitamoto et al. (1990) estimated $E(B-V) \sim 1\left(N_{\mathrm{H}} \sim 5.3 \times 10^{21} \mathrm{~cm}^{-2}\right)$, and the extinction estimated from the optical spectra obtained by Casares et al. $(2004,2009)$ is consistent with this value.

As the measured spectrum is intrinsically soft, the differing extinction estimates result in the unabsorbed flux for the powerlaw model being approximately 1.5 times that measured using the bremsstrahlung model. The power-law model is formally a 


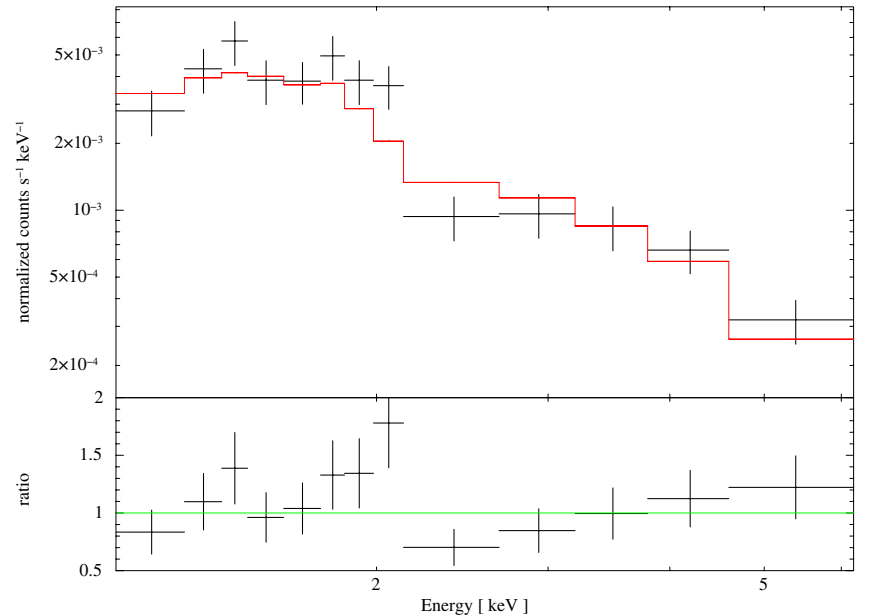

Figure 2. Chandra 1-6 keV spectrum of GS 1354-64. The exposure time is $\sim 39 \mathrm{ks}$. The best fit power-law model $(\Gamma \sim 2.1)$ is plotted, the source luminosity is $L_{x} \sim 0.7-6 \times 10^{34} \mathrm{erg} \mathrm{s}^{-1}$, see Table 1 for details.

(A color version of this figure is available in the online journal.)

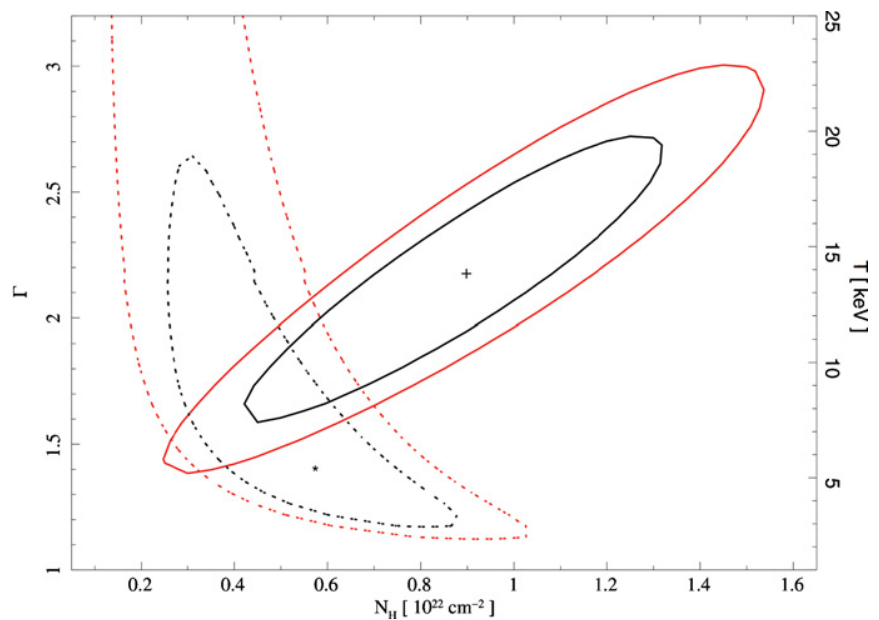

Figure 3. Contour plots corresponding to the best fit models in Table 1. The power-law fit is represented by the solid lines, while the thermal bremsstrahlung model is represented by the dotted contours. Contours plotted represent the $68 \%$ and $90 \%$ confidence levels.

(A color version of this figure is available in the online journal.)

better fit to the observed spectrum; however, the low number of bins render this insignificant.

In Figure 4, we plot the luminosity calculated using the unabsorbed flux from the best fit power-law model in Table 1 and the distance estimates of Casares et al. (2009), i.e., $25 \leqslant$ $d_{\mathrm{kpc}} \leqslant 61$. GS 1354-64 is observed to be at a luminosity over an order of magnitude greater than that expected from a quiescent black hole. For this system to be consistent with the known distribution of black holes would require a decrease of the distance to $\sim 5.8 \mathrm{kpc}$. Monitoring observations at optical wavelengths with the Faulkes telescope ${ }^{1}$ revealed GS 1354-64 to be consistent with the known quiescent optical flux at the time of our Chandra observations, though, we note the nearest observation took place approximately 2 months prior to the Chandra observation described herein. While observations with the available X-ray, all sky monitors show no evidence for increased activity either before or after our observation.

\footnotetext{
1 http://staff.science.uva.nl/ davidr/faulkes/ (Lewis et al. 2008)
}

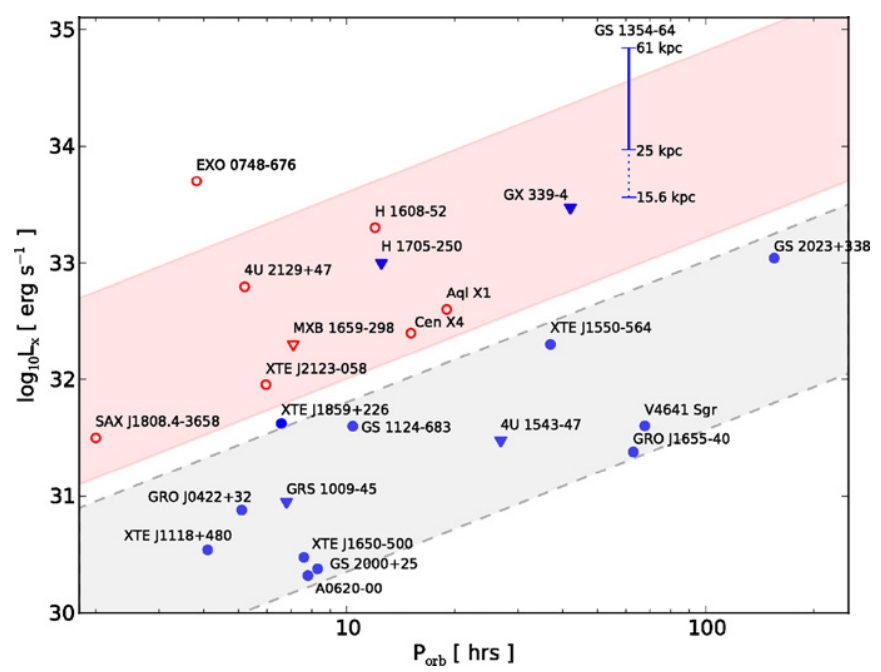

Figure 4. Vertical blue line indicates the measured luminosity of GS 1354-64 assuming a distance of $25 \mathrm{kpc} \leqslant d \leqslant 61 \mathrm{kpc}$ (Casares et al. 2009), while the dotted extension indicates the estimated lower limit to the distance, see Section 4.1 for details. GS 1354-64 is observed to be over an order of magnitude too luminous in comparison to the known distribution of quiescent black holes (solid blue symbols). The open red symbols indicate the quiescent luminosities of neutron stars. Upper limits are represented by triangles. The red and blue filled areas indicate the regions in the $L_{x}, P_{\text {orb }}$ plane occupied by the neutron star and black holes systems, respectively. Black hole luminosities are from Garcia et al. (2001); Kong et al. (2002); Sutaria et al. (2002); Tomsick et al. (2003); Hameury et al. (2003); McClintock et al. (2004); Corbel et al. (2006); Gallo et al. (2008), and the neutron star luminosities are from Garcia et al. (2001); Tomsick et al. (2004); Lin et al. (2009); Degenaar et al. (2011).

(A color version of this figure is available in the online journal.)

However, a faint outburst would not have been detected by these observations.

There are two other stellar mass black holes with weak quiescent luminosity constraints. The first system, H 1705250, had only been observed by ROSAT. This upper limit will be updated soon by Chandra (Obsid: 11041, PI: Kong). The second system, GX 339-4, is an extremely active transient having exhibited five large and $\sim 10$ small outburst in the last 15 years. The most sensitive observation was obtained by Chandra in 2003 and revealed this system at the faintest level measured to date. However, this observation occurred $\sim 10$ months after the 2002 outburst and 7 months prior to the 2003/2004 outburst (Gallo et al. 2003). Hence, it is highly unlikely that GX 339-4 was in quiescence at the time of this observation. The current upper limits for these systems are represented by the solid triangles in Figure 4.

\section{DISCUSSION}

We have observed the quiescent stellar mass black hole GS 1354-64 with Chandra and found it to be at a luminosity of $\sim 10^{34} \mathrm{erg} \mathrm{s}^{-1}$. This is over an order of magnitude greater than expected. Here, we discuss the uncertainty in the luminosity estimate and consider the implications of this measurement for the nature of the accretion flow in the quiescent state.

\subsection{Distance to GS 1354-64}

In order to compare to the other systems in Figure 4, we must know the distance to each system. Casares et al. $(2004,2009)$ have calculated the minimum distance to GS $1354-64$ to be $d_{\min } \geqslant 25 \mathrm{kpc}$. The distance estimate depends on the known spectral type of the secondary star, the apparent magnitude of the star, the contribution from the accretion disk, and the 
extinction. The extinction is the most uncertain quantity in the distance calculation. Casares et al. (2004) estimate the extinction from known empirical relationships between observed spectral features and the column density resulting in an estimated $E(B-V) \sim 1$, or more specifically $E(B-V) \geqslant 0.78$, consistent with the estimate of Kitamoto et al. (1990). However, the measured neutral hydrogen column in this field suggests a larger value, i.e., $N_{\mathrm{H}} \sim 7.27 \times 10^{21} \mathrm{~cm}^{-2}$ (Kalberla et al. 2005) or $E(B-V) \sim 1.4$.

A higher level of extinction will effect the estimated minimum distance to this system of $d_{\min } \geqslant 25 \mathrm{kpc}$, in particular for a column density of $N_{\mathrm{H}} \sim 7.27 \times 10^{21} \mathrm{~cm}^{-2}$ we find a minimum distance of $d_{\min } \geqslant 17.8 \mathrm{kpc}$, decreasing to $10.2 \mathrm{kpc}$ for a column of $10^{22} \mathrm{~cm}^{-2}$. The empirical diffuse interstellar band relation of Herbig (1975) as used in Casares et al. (2004) suggests an upper limit to the reddening of $E(B-V) \leqslant$ $1.5\left(N_{\mathrm{H}} \sim 7.9 \times 10^{21} \mathrm{~cm}^{-2}\right)$ corresponding to a distance of $\sim 15.6 \mathrm{kpc}$. Nonetheless, even in the unlikely event that the extinction is underestimated, GS 1354-64 remains too luminous in comparison to the known distribution.

\subsection{Possibilities}

The measured X-ray luminosity allows one to estimate the mass accretion rate, we do this under two assumptions for the accretion efficiency, i.e., $L_{x}=\eta \dot{M} c^{2}$. Assuming standard thin disk accretion $(\eta=0.1)$ implies an accretion rate of $10^{-11}\left(L_{x} / 10^{34}\right) M_{\odot} \mathrm{yr}^{-1}$, whereas for an ADAF, an efficiency of $\eta=10^{-2}$ to $10^{-4}$ is expected, and hence, a commensurably larger accretion rate. Accretion rates onto the outer disk of $\sim 10^{-10} M_{\odot} \mathrm{yr}^{-1}$ have been estimated for a number of quiescent BHBs, e.g., A0620-00 (McClintock et al. 1995) and XTE J1550-564 (Orosz et al. 2011). As such, the accretion rates necessary to generate the observed luminosity appear to be feasible.

We also note the similarity between the observed X-ray variability of a factor of $\sim 2$ (see Figure 1) and that typically observed at optical wavelengths, where variability of approximately 1 magnitude $(\sim 2.5 \times$ in linear units) is common from GS 1354-64 in quiescence (Casares et al. 2004, 2009). Correlated optical/X-ray variability has been observed from V404 $\mathrm{Cyg}$ in quiescence. However, the origin of this variability is not understood, with emission from the inner ADAF and/or irradiation of the disk being likely (Hynes et al. 2004). A similar scenario is likely here, in the case of GS 1354-64; however, further observations are required to confirm this possibility.

Below we briefly discuss what we consider to be the most likely explanation for the large observed quiescent luminosity.

1. A temporary increase in the mass accretion rate, $\dot{M}$. For example, observations of the stellar mass black hole GRO J1655-40 ( $P_{\text {orb }} \sim 63 \mathrm{hr}$ ) have revealed luminosity variations by a factor of 10 (Hameury et al. 2003). However, the brighter of these observations was obtained $\sim 9$ months after the 1995 outburst and only one month prior to the 1996 outburst, i.e., the observed large luminosity was caused by the system not being quiescent at the time of the $A S C A$ observations. Long-term variations of a factor of $\sim 10$ have also been observed from V404 Cyg $\left(P_{\text {orb }} \sim 155 \mathrm{hr}\right.$; Bradley et al. 2007), while variations of $\sim 20 \times$ have been observed on kilo-second timescales with Chandra (Hynes et al. 2004). However, in both cases the luminosity remained consistent with that expected for the distribution of quiescent black holes (see Figure 4). Observations of
Sgr $\mathrm{A}^{*}$ have revealed flares with an amplitude of greater than 100 times the true quiescent rate (Baganoff et al. 2001; Porquet et al. 2008), but these flares typically have a duration of a few hours and as such are unlikely the cause of the large luminosity, which we observe from GS 1354-64.

2. The actual quiescent accretion luminosity from this system is $\gtrsim 10^{34} \mathrm{erg} \mathrm{s}^{-1}$. This would be the largest Eddington scaled luminosity measured from a black hole in quiescence to date, and would point to the existence of a unique lowluminosity accretion flow in this system. Standard accretion disk theory predicts the disk to be truncated, with a lowmass accretion rate and luminosity (McClintock et al. 1995; Narayan et al. 1996; Lasota 2008), e.g., both GRO J1655-40 $\left(M_{x} \sim 6.5 M_{\odot}, P_{\text {orb }} \sim 63 \mathrm{hr}\right)$ and V4641 $\operatorname{Sgr}\left(M_{x} \sim\right.$ $7 M_{\odot}, P_{\text {orb }} \sim 68 \mathrm{hr}$ ) have similar orbital periods to GS 1354-64 $\left(M_{x} \gtrsim 7 M_{\odot}, P_{\text {orb }} \sim 60 \mathrm{hr}\right)$, but have quiescent luminosities consistent with the other black holes, i.e., $L_{x} \sim 5.9 \times 10^{31} \mathrm{erg} \mathrm{s}^{-1}$ and $4 \times 10^{31} \mathrm{erg} \mathrm{s}^{-1}$, respectively (Hameury et al. 2003; Tomsick et al. 2003). The nature of the mass donor secondary star is also sufficiently similar with F4III-IV (Orosz \& Bailyn 1997) and B9III (Orosz et al. 2001), respectively, in comparison to the G0-G5III secondary in GS 1354-64. If the large quiescent luminosity we have measured is confirmed, it would suggest the existence of a previously unrecognized stable mode of low-luminosity accretion $\left(L_{x} \sim 10^{-5} L_{\text {Edd }}\right.$ ), and with it a population of relatively faint accreting black holes in the galaxy, e.g., Menou et al. (1999).

\subsection{BH versus NS Luminosities}

A comparison of the quiescent luminosities of the black hole and neutron star binaries revealed the black hole systems to be a factor of $\sim 100$ times fainter (Garcia et al. 2001; Figure 4). This has been interpreted as evidence for the absence of a solid surface in the black hole systems, and conversely as indirect evidence for the existence of the event horizon. The observation presented of GS 1354-64 herein contradicts this empirical relationship. Previously, an observation of the neutron star binary $1 \mathrm{H} 1905+00$ was claimed to also contradict this relationship (Jonker et al. 2007); however, due to its ultra-compact nature, it is not clear that the accretion flow in this system is comparable to that in the BH and NS systems in Figure 4 (Lasota 2008). If the measured luminosity of GS 1354-64 (Figure 4) is shown to be stable by future observations, one could ask if the observed difference between the black hole and neutron stars in quiescence is not caused by the absence of a surface in the black hole systems but instead by the favoring of this higher luminosity quiescent accretion flow in the neutron star systems.

We thank Dave Russell for discussions regarding the Faulkes monitoring of this system. M.T.R. and J.M.M. gratefully acknowledge support through the Chandra Guest Observer program. This research made use of the SIMBAD database, operated at CDS, Strasbourg, France and NASA's Astrophysics Data System. We thank the anonymous referee for useful comments.

\section{REFERENCES}

Baganoff, F. K., et al. 2001, Nature, 413, 45

Blandford, R., \& Begelman, M. 1999, MNRAS, 303, 1

Bradley, C. K., Hynes, R. I., Kong, A. K. H., Haswell, C. A., Casares, J., \& Gallo, E. 2007, ApJ, 667, 427 
Brocksopp, C., Jonker, P. G., Fender, R. P., Groot, P. J., van der Klis, M., \& Tingay, S. J. 2001, MNRAS, 323, 517

Casares, J., Zurita, C., Shahbaz, T., Charles, P. A., \& Fender, R. P. 2004, ApJ, 613,133

Casares, J., et al. 2009, ApJS, 181, 238

Corbel, S., Tomsick, J. A., \& Kaaret, P. 2006, ApJ, 636, 971

Corral-Santana, J. M., Casares, J., Shahbaz, T., Zurita, C., Martnez-Pais, I. G., \& Rodrguez-Gil, P. 2011, MNRAS, 413, 15

Crowther, P. A., Barnard, R., Carpano, S., Clark, J. S., Dhillon, V. S., \& Pollock, A. M. T. 2010, MNRAS, 403, 41

Degenaar, N., et al. 2011, MNRAS, 412, 1409

Doeleman, S. S., et al. 2008, Nature, 455, 78

Fender, R. P., Gallo, E., \& Jonker, P. G. 2003, MNRAS, 343, 99

Gallo, E., Fender, R., \& Corbel, S. 2003, ATel, 196

Gallo, E., Fender, R. P., Miller-Jones, J. C. A., Merloni, A., Jonker, P. G., Heinz, S., Maccarone, T., \& Van der Klis, M. 2006, MNRAS, 370, 1351

Gallo, E., Homan, J., Jonker, P. G., \& Tomsick, J. A. 2008, ApJ, 683, 51

Garcia, M. R., McClintock, J. E., Narayan, R., \& Callanan, P. J. 1998, in ASP Conf. Ser. 137, Wild Stars in the Old West, ed. S. Howell, E. Kuulkers, \& C. Woodward (San Francisco, CA: ASP), 506

Garcia, M. R., McClintock, J. E., Narayan, R., Callanan, P., Barret, D., \& Murray, S. 2001, ApJ, 553, 47

Hameury, J. M., Barret, D., Lasota, J. P., McClintock, J. E., Menou, K., Motch, C., Olive, J. F., \& Webb, N. 2003, A\&A, 399, 631

Herbig, G. H. 1975, ApJ, 196, 129

Hynes, R. I., et al. 2004, ApJ, 611, 125

Jonker, P. G., Steeghs, D., Chakrabarty, D., \& Juett, A. M. 2007, ApJ, 665, 147

Kalberla, P. M. W., Burton, W. B., Hartmann, D., Arnal, E. M., Bajaja, E., Morras, R., \& Pöppel, W. G. L. 2005, A\&A, 440, 775

Kalogera, V., \& Baym, G. 1996, ApJ, 470, 61

Kitamoto, S., Tsunemi, H., Pedersen, H., Ilovaisky, S. A., \& van der Klis, M. 1990, ApJ, 361, 590
Kong, A. K. H., McClintock, J. E., Garcia, M. R., Murray, S. S., \& Barret, D. 2002, ApJ, 570, 277

Lasota, J. P. 2008, New Astron. Rev., 51, 752

Lewis, F., Roche, P., Russell, D. M., \& Fender, R. P. 2008, in AIP Conf. Proc. 1010, A Population Explosion: The Nature \& Evolution of X-ray Binaries in Diverse Environments, ed. R. M. Bandyopadhyay et al. (Melville, NY: AIP), 204

Lin, J., Nowak, M. A., \& Chakrabarty, D. 2009, ApJ, 706, 1069

Makino, F. 1987, IAU Circ., 4342

McClintock, J. E., Horne, K., \& Remillard, R. A. 1995, ApJ, 442, 358

McClintock, J. E., Narayan, R., \& Rybicki, G. B. 2004, ApJ, 615, 402

McClintock, J. E., \& Remillard, R. A. 2006, in Compact Stellar X-Ray Sources, ed. W. H. G. Lewin \& M. van der Klis (Cambridge: Cambridge Univ. Press), 157

Menou, K., Narayan, R., \& Lasota, J. P. 1999, ApJ, 513, 811

Narayan, R., Garcia, M. R., \& McClintock, J. E. 1997, ApJ, 478, 79

Narayan, R., \& McClintock, J. E. 2008, New Astron. Rev., 51, 733

Narayan, R., McClintock, J. E., \& Yi, I. 1996, ApJ, 457, 821

Orosz, J. A., \& Bailyn, C. D. 1997, ApJ, 477, 876

Orosz, J. A., et al. 2001, ApJ, 555, 489

Orosz, J. A., McClintock, J. E., \& Narayan, R. 2007, Nature, 449, 872

Orosz, J. A., Steiner, J. F., McClintock, J. E., Torres, M. A. P., Remillard, R. A. Bailyn, C. D., \& Miller, J. M. 2011, ApJ, 730, 75

Porquet, D., et al. 2008, A\&A, 488, 549

Rhoades, C. E., \& Ruffini, R. 1974, Phys. Rev. Lett., 32, 324

Silverman, J. M., \& Filippenko, A. V. 2008, ApJ, 678, 17

Sutaria, F. K., et al. 2002, A\&A, 391, 993

Tomsick, J. A., Gelino, D. M., Halpern, J. P., \& Kaaret, P. 2004, ApJ, 610, 933

Tomsick, J. A., et al. 2003, ApJ, 597, 133

Voges, W., et al. 1999, A\&A, 349, 389

Yuan, F., Quataert, E., \& Narayan, R. 2003, ApJ, 598, 301

Yuan, F., Yu, Z., \& Ho, L. C. 2009, ApJ, 703, 1034

Yusef-Zadeh, F., et al. 2009, ApJ, 704, 348 